\title{
POTENTIAL OF LEADING INDICATOR DATA COLLECTION AND ANALYSIS FOR PROXIMITY DETECTION AND ALERT TECHNOLOGY IN CONSTRUCTION
}

\author{
Eric D. Marks ${ }^{1}$, Jeremy E. Wetherford ${ }^{1}$, ${ }^{*}$ Dr. Jochen Teizer $^{1}$, and Dr. Nobuyoshi Yabuki ${ }^{2}$ \\ (*Corresponding author: teizer@gatech.edu) \\ ${ }^{1}$ RAPIDS Construction Safety and Technology Laboratory \\ School of Civil and Environmental Engineering \\ Georgia Institute of Technology \\ 790 Atlantic Drive N.W. \\ Atlanta, GA 30332-0355, U.S.A. \\ Phome (404) 894-8260 \\ ${ }^{2}$ Division of Sustainable Energy and Environmental Engineering \\ Graduate School of Engineering \\ Osaka University, Japan
}




\title{
POTENTIAL OF LEADING INDICATOR DATA COLLECTION AND ANALYSIS FOR PROXIMITY DETECTION AND ALERT TECHNOLOGY IN CONSTRUCTION
}

\begin{abstract}
Fatalities resulting from ground workers colliding with objects and construction equipment accounted for approximately $18 \%$ of the total construction fatalities experienced in 2010. Current injury and fatality measuring methods have proven to be inadequate due to their use of passive measures of safety rather than a more active approach, such as the use of leading indicator data (also called near misses) of hazardous proximity situations between ground workers and heavy equipment on construction sites. The objectives are to create and evaluate the effectiveness of a reliable algorithm for collecting and analyzing the resulting leading indicator data from hazardous proximity situations. Numerous experiments emulating typical interactions between workers-on-foot and heavy equipment are used to evaluate the proximity sensing and detection technology. Safety leading indicator data (also called near misses) generated by the proximity sensing and detection technology during the experimental trials are used to create and assess the resulting algorithm. Results from the experiments indicate that leading indicator data generated from these systems can be processed and analyzed to identify hazardous proximity situations and incidents on construction sites.
\end{abstract}

\section{KEYWORDS}

Construction workers-on-foot; equipment operator visibility; hazardous proximity situations; heavy construction equipment; leading safety indicators; proximity detection, warning, alert technology; safety.

\section{INTRODUCTION}

Characteristics of construction sites including their dynamic nature often require multiple construction resources (ground workers, construction equipment, and materials) to operate in close proximity to one another. When heavy construction equipment is operating in close proximity to ground workers, hazardous proximity working conditions are created. These conditions can result in property damage, worker injuries, and worker fatalities. A majority of the research efforts are used to gather and analyze injury and fatality statistics of contact collisions between ground workers and heavy construction equipment. Previous research has also focused on investigating root causes of accidents between heavy construction equipment and ground workers, but has not implemented technology as a potential solution (Pratt et al. 2001).

Construction safety research currently lacks scientific evaluation data and results gathered through experimentation of safety technologies including proximity warning and alert systems (Teizer et al. 2010, Marks and Teizer 2012). These systems provide alerts in real-time when heavy construction equipment is in too close proximity to ground workers. Output data from these systems can be analyzed to evaluate the safety performance of construction personnel with regards to hazardous proximity conditions. An algorithm was developed to transform output data from these systems to safety leading indicator information. Experimental trials designed to emulate conditions typical of construction sites are required to evaluate the reliability and effectiveness of the resulting safety leading indicator information.

\section{LITERATURE REVIEW}

Construction sites typically offer limited operating space for construction resources (including workers and equipment). These site conditions cause heavy construction equipment and ground workers to function in close proximity to one another resulting in hazardous working conditions. The following review covers construction industry safety statistics, proximity detection and alert systems, and safety leading indicator research. 


\section{Construction Industry Statistics}

The construction industry continues to rank as one of the highest for workplace fatalities per year. The industry recorded 721 fatalities in 2011, $17 \%$ of which resulted from workers colliding with objects or equipment (CFOI 2011). In the same year, fatalities caused by collisions between workers and objects or equipment accounted for $2.6 \%$ of all workplace fatalities experienced in the US. Since 2002, the construction industry has averaged 197 fatalities per year resulting from workers being struck-by equipment or objects (CFOI 2009).

Injuries and illnesses related to proximity issues on construction sites also present safety concerns for workers. The private sector of the construction industry recorded 30,330 injuries and illnesses caused by ground workers colliding with construction equipment and other objects (BLS 2009). These injuries accounted for $33 \%$ of all construction worker injuries and illnesses in that year. All recorded injuries and illnesses are limited to accidents involving personnel who were absent from work as a result of the incident.

\section{Proximity Detection and Alert Technology}

Many technologies including RADAR (Radio Detection and Ranging), Global Positioning System (GPS), sonar, vision, radio transceiver tags, magnetic marking fields, etc. are thought to be capable of alerting construction personnel in real-time when hazardous proximity conditions exist. Ruff (2005) found that each of these technologies has a unique set of limitations when deployed on a construction site including availability of signal strength, size, weight, power source, alert method, precision, reliability, and alert distance. Many of these limitations were assessed when the systems were deployed in a simulated construction environment and active (battery-powered) radio frequency (RF) technology demonstrated an ability to satisfy many of these parameters (Teizer et al. 2010).

\section{Safety Leading Indicators}

One research effort evaluated the different metrics and measures for evaluating safety performance on construction sites including leading indicators such as near miss events. Results indicate that leading indicators can enhance the current metrics and measured used to evaluate safety performance (Hinze and Godfrey 2003). Another study defined leading indicators as "measures of attitudes, behaviors, practices, or conditions that influence construction safety performance" (Hallowell and Hinze 2012). The study further divided leading indicators into passive (safety strategies that should be implemented before construction begins) and active (safety-related practices that can be measured during the construction phase). A method for measuring safety of struck-by accidents was created using the following steps: (1) Identify safety attributes, (2) determine frequency and severity, and (3) determine risk through frequency and severity. Several industries such as firefighting, nursing and the airlines maintain a database of reported safety leading indicators such as near miss events (FSF 2012; FEMA 2012; Henneman and Gawlinski 2004).

However, a lack of scientific evaluation data exists for automated safety technology for construction sites. Furthermore, research effort is required to develop algorithms to generate safety leading indicator information from current safety databases. This evaluation of safety technologies and creation of an algorithm should be accomplished through current or newly developed experimental methods, case studies, and data analyses.

\section{OBJECTIVE AND SCOPE}

The objective is to create an algorithm to generate safety leading indicator information by analyzing output databases of proximity warning and alert systems. The functionality of these systems provides an alert in real-time when ground workers are in too close proximity to heavy construction equipment. The purpose of the algorithm and resulting information is to analyze and generate safety leading indicator data for each ground worker near heavy construction equipment. Experiments were designed to assess the reliability and effectiveness of the created algorithm in simulated construction 
environment conditions. Experimental trials implemented a commercially-available proximity warning and alert system using radio frequency $(\mathrm{RF})$ technology and were performed at grade.

\section{METHODOLOGY AND RESULTS}

All experimental designs and trials were intended to generate proximity alert distance data between construction equipment and ground workers in a simulated construction site environment. These trials provided the input data to evaluate the reliability and effectiveness of the developed algorithm for analyzing safety leading indicator data for hazardous proximity situations. The technology used, performed experimental trials, and resulting outputs of the algorithm are discussed.

\section{Proximity Warning and Alert Technology}

A proximity warning and alert system that uses radio frequency (RF) technology (Ultra High Frequency) was implemented for the experimental trials and development of the algorithm functions. If two or more construction resources are in too close proximity, the sensing technology will activate an alert to warn construction personnel. The system has two major components:

(1) Equipment Protection Unit (EPU): Construction equipment device comprised of a single directional antenna, reader, and alert mechanism. It can be mounted and connected to the main power source of the equipment

(2) Personnel Protection Unit (PPU): Personal device mounted on safety equipment of ground workers comprised of a chip, battery, and signal receiver/ transmitter

A signal is broadcasted by the EPU's antenna and is intercepted by the PPU when the devices are in too close proximity defined by the system's alert distance. The EPU antenna should be positioned on the construction equipment such that the line-of-sight between the EPU and PPU is not obstructed. The signal is broadcasted in a directional manner and covers approximately 60 degrees on either side of a centerline perpendicular to the face plane of the antenna. When a proximity breach is detected, the PPU intercepts the radio signal and immediately returns a signal to the EPU which triggers an audible alert automatically in real-time. This alert is different from other sounds and back-up alerts common to construction sites.

The proximity warning and alert system is also capable of logging data concerning proximity breaches between construction equipment and ground workers. The system records a timestamp for each proximity breach, the PPU identification number, the EPU antenna involved, and the magnitude of the reflected radio frequency signal as the Received Signal Strength Indication (RSSI). The system evaluates and records the status approximately four times per second. This information was used as input data for the developed algorithm.

\section{Leading Indicator Data Collection}

Outputs of the proximity warning and alert system (including the timestamp of the proximity breach, received signal strength indication, ground worker ID, and equipment antenna ID) provided data to calculate safety leading indicator information for hazardous proximity situations. The leading indicators monitored were the amount of time a ground worker spends within the alert radius and the total number of recorded proximity breaches. Because the output data is generated in real-time, the leading indicator information can be analyzed and be available for safety managers at the end of the workday (or if transmitted from the equipment wirelessly almost in real-time). Output data from the proximity warning and alert system is available in a text file. This text file is inserted into a Microsoft Excel ${ }^{\mathrm{TM}}$ file for readability purposes that practitioners can use. However, the platform used for the analysis was MATLAB $^{\mathrm{TM}}$. Several programs were written in MATLAB ${ }^{\mathrm{TM}}$ to complement the data analysis effort. 


\section{Leading Indicator Data Analysis}

Each of the calculated statistics provides a useful tool in determining the safety performance of a ground worker near heavy construction equipment. The key statistics for each ground worker were defined as the number of recorded proximity breaches and the time spent within the pre-defined alert radius. The number of worker proximity breaches in conjunction with the total duration spent within the proximity alert zone reveals the frequency and duration a worker is exposed to hazardous proximity conditions on construction sites. By monitoring these key statistics through the employment of the two programs, it is theorized that intervention may be recommended to alter a ground workers' behaviors around heavy construction equipment.

Because the initial text file is difficult to read and provides little usefulness in its raw state due to the vast amount of timestamps, the first of the two programs was written in order to refine the data and output the key statistics for all workers for a given workday. The initial program uses the raw text (CSV) file from a given workday as its sole input and iterates through each timestamp of the file. During these iterations, which are completed for each worker, both the frequency of proximity breaches and the duration of exposure to hazardous proximity conditions for each worker are continually cumulated. To calculate the duration of a worker inside the proximity alert range, the program compares the current timestamp with the previous timestamp. If the timestamp differs, a second is added to the ongoing counter. This is done instead of taking the difference in the timestamps because the proximity warning and alert system records timestamps several times per second. If two timestamps have a difference of more than one second, it indicates that the worker exited the proximity alert zone or the range of a particular antenna. A proximity breach is added to the incident counter, if more than one second difference exists. The number of recorded proximity breaches is determined by counting the number of timestamps associated with each ground worker. The result is the number of proximity breaches and the time spent inside the proximity. Separate time counters exist for each antenna as well as one the total time counters for the system.

The analysis algorithm is also capable of identifying individual ground workers from their PPU identifications and pieces of heavy construction equipment from their EPU identifications. A simple switch statement can be written to replace worker identifications with actual worker names and important details such as worker safety history, training/certification levels, and work experience. The same information can be included with equipment identification information. Upon determining the key group of statistics for each ground worker, the program outputs the refined data into a user-friendly separate Excel ${ }^{\mathrm{TM}}$ file.

A second program was written to monitor trends in hazardous proximity conditions by determining the percent change for key statistics of an individual worker between different workdays. The program uses the two refined data files outputted from the first program and iterates through each of the files to calculate the percent change for each worker on a daily basis. The developed data mining algorithm in MATLAB ${ }^{\mathrm{TM}}$ then outputs a separate user-friendly Excel ${ }^{\mathrm{TM}}$ file with the percent changes recorded. The program can be modified to measure percent change from a larger time difference, such as weekly monitoring. By monitoring the percent changes in the key statistics, interventions can be recommended to curve safety behavior of construction workers.

\section{Experiments}

The experiments were designed to assess the reliability and effectives of the developed algorithm. All experimental trials were performed in an outdoor environment with mostly clear weather conditions and a temperature of 65 degrees Fahrenheit. A clear and flat grass ground surface with no obstructions was used as a test bed for these trials.

The EPU's antenna component was mounted on a tripod with the face plane perpendicular to the ground surface for the experimental trials. The antenna's centroid was positioned 1.15 meters vertically from the ground surface. This vertical represents the average distance between the top of the test person's hard hat and the center of the test person's vest. The test person equipped with a semi-passive Radio 
Frequency Identification (RFID) tag for the PPU remained in a stationary position approximately 5 meters from the EPU. Figure 1 shows the test bed.
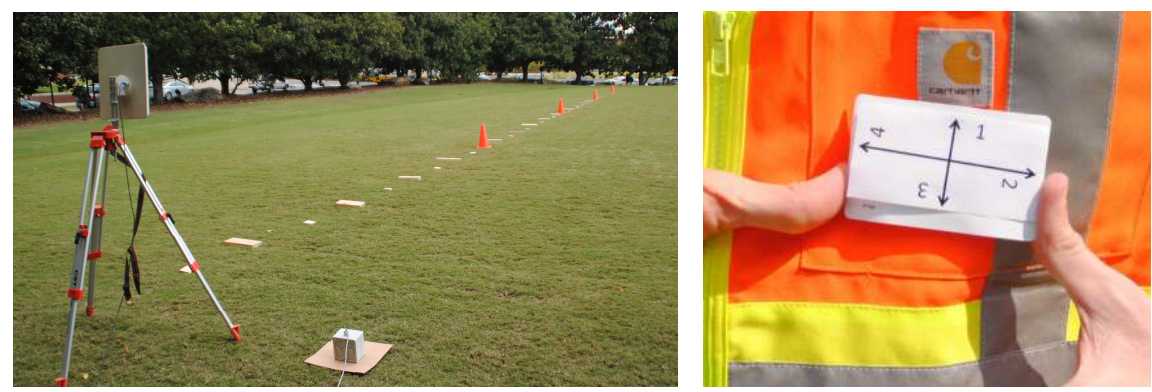

Figure 1 - Proximity detection and alert system components: EPU (left) and PPU (right)

\section{Proximity Breach Duration}

A total of three experimental trials were performed to evaluate the created algorithm's reliability and effectiveness for duration of proximity breaches. A description of each set of experimental trials is listed:

Trial 1 Stationary individual PPU: A test person equipped with a semi-passive RFID tag in a safety vest for a PPU remained in a static position five meters from the EPU's antenna along a centerline path for 30 seconds for three individual trials using three different PPU's.

Trial 2 Multiple stationary PPU's: A test person equipped with three semi-passive RFID tags in a safety vest for a PPU remained in a static position five meters from the EPU's antenna along a centerline path for 30 seconds.

Trial 3 Percent change of multiple PPU's: A test person equipped with three semi-passive RFID tags in a safety vest for a PPU remained in a static position five meters from the EPU's antenna along a centerline path for 30 seconds for one trial and 20 seconds for a second trial.

Results from each of the three trials are summarized in Table 1. The PPU identification number is given, the analyzed duration in the hazardous proximity detection zone, and the percent error from the known duration.

Table 1 - Calculated versus actual duration of each experimental trial

\begin{tabular}{lcccc}
\hline & PPU ID & \multicolumn{2}{c}{ Duration (in seconds) } & Percent Error \\
\hline Trial 1 & & Calculated & Actual & \\
& Tag \#1 & 32 & 30 & $+6.7 \%$ \\
& Tag \#2 & 32 & 30 & $+6.7 \%$ \\
Trial 2 & Tag \#3 & 32 & 30 & $+6.7 \%$ \\
& & & & \\
& Tag \#1 & 33 & 30 & $+10.0 \%$ \\
& Tag \#2 & 31 & 30 & $+3.3 \%$ \\
Trial 3 & Tag \#3 & 34 & 30 & $+13.3 \%$ \\
& & & & \\
& Tag \#1 & $33.3 \%$ & $33.3 \%$ & $0.0 \%$ \\
& Tag \#2 & $32.3 \%$ & $33.3 \%$ & $-3.0 \%$ \\
& Tag \#3 & $35.3 \%$ & $33.3 \%$ & $+6.0 \%$ \\
\hline
\end{tabular}


When testing the timed duration (30 seconds) for each tag individually, the algorithm output was 32 minutes which resulted in a $6.7 \%$ error for all tests in trial 1 . This indicates systematic error is present along with the assumed random error of using humans to measure the known time trials. Of the three trials, trial 1 performed with the strongest correlation between the calculated and actual durations. For the second trial, all timed measurements were above the known 30 seconds, and two tags recorded an error of ten percent or higher. All tags in trial 3 performed with an error of six percent or lower. Both cases where the calculated percent change was not the actual value in trial 3, the percent change values were greater than the actual value.

\section{Frequency of Proximity Breaches}

Two experimental trials were deployed to evaluate the created algorithm's reliability and effectiveness for the frequency of proximity breaches. A description of each set of experimental trials is listed:

Trial 1 Stationary individual PPU: A test person equipped with a semi-passive RFID tag in a safety vest for a PPU remained in a static position five meters from the EPU's antenna along a centerline path for five seconds for three individual trials using three different PPU's. The stationary proximity breaches were repeated five times for each PPU tested.

Trial 2 Multiple stationary PPU's: A test person equipped with three semi-passive RFID tags in a safety vest for a PPU remained in a static position five meters from the EPU's antenna along a centerline path for five seconds. After the stationary five seconds, the test person exited the proximity detection zone for five, ten, and fifteen second intervals before re-entering. These steps were repeated five times for each set of timed intervals of proximity breaches.

Results from the two trials are shown in Table 2. The PPU identification number is given, the analyzed frequency in the hazardous proximity detection zone, and the percent error from known five frequencies for each trial.

Table 2 - Calculated versus actual frequency of breaches of each experimental trial

\begin{tabular}{cccc}
\hline & PPU ID & Interval (in seconds) & Number of breaches \\
\hline Trial 1 & Tag \#1 & 5 & 5 \\
& & 10 & 5 \\
& & 15 & 5 \\
& Tag \#2 & 5 & 4 \\
& & 10 & 5 \\
& & 15 & 5 \\
& Tag \#3 & 5 & 4 \\
& & 10 & 5 \\
Trial 2 & Tag \#1 & 15 & 5 \\
& & 5 & 5 \\
& & 10 & 5 \\
& Tag \#2 & 15 & 4 \\
& & 5 & 5 \\
& & 10 & 4 \\
& Tag \#3 & 15 & 5 \\
& & 5 & 5 \\
& & 10 & 5 \\
& & 15 & 5 \\
\hline
\end{tabular}


The algorithm was able to identify and record majority of the proximity breaches. Of the 90 known proximity breaches, four proximity breaches were not recorded $(4.4 \%)$. There was no correlation between the instances where a proximity breach failed to record. Tag 2 experienced two failed recordings of proximity breaches, whereas tag 1 and 3 each experienced one case. Two of the failed recording of proximity breaches occurred during a five second interval, one case occurred in a ten second interval and the other in a 15 second interval. Two failed recordings of proximity breaches occurred during the individual PPU trials while the other two cases occurred during multiple PPU trials.

\section{CONCLUSIONS}

Contact collisions between ground workers and heavy construction equipment are one of the leading causes of fatalities within the construction industry. The purpose of this research was to create and evaluate the effectiveness of an algorithm designed to generate safety leading indicator information. The algorithm functions in conjunction with a semi-passive radio frequency proximity detection and alert system. Further research is required to implement the developed hardware and data mining algorithms for long-term performance measurement in the field. More understanding is also needed to better understand which other safety leading indicators (such as distance of the proximity breach or location of the worker in relation to the construction equipment) should be captured and analyzed through the algorithm. By allowing safety managers on construction sites to have access to safety leading indicator data, safety training and education for workers can be enhanced for hazardous proximity conditions between ground workers and heavy construction equipment.

\section{REFERENCES}

Bureau of Labor Statistics (2010). Census of Fatal Occupational Injuries (CFOI) - Current and Revised Data. Retrieved from the Bureau of Labor Statistics website: http://www.bls.gov/iff/oshcfooil.htm\#2007

Bureau of Labor Statistics (2009). Census of Fatal Occupational Injuries (CFOI) - Current and Revised Data. Retrieved from the Bureau of Labor Statistics website: http://www.bls.gov/iff/oshcfooil.htm\#2008

Census of Fatal Occupational Injuries (2011). Fatal Occupational Injuries by Industry and Selected Event or Exposure. Retrieved from the Bureau of Labor Statistics, http://www.bls.gov/iff/oshcfoil.htm

Census of Fatal Occupational Injuries (2009). Fatal Occupational Injuries by Industry and Selected Event or Exposure. Retrieved from the Bureau of Labor Statistics, http://www.bls.gov/iff/oshcfoil.htm

Federal Emergency Management Agency (2012). National Fire Fighter - Near Miss Reporting System. Retrieved from the National Fire Fighter website: http://www.firefighternearmiss.com/index.php/near-miss-partners

Flight Safety Foundation (2012). Aviation Safety Events. Retrieved from the Flight Safety Foundation website: http://flightsafety.org/ap_home.html

Hallowell, M., and Hinze, J. (2012). "Measuring Safety Performance with Active Safety Leading Indicators." Construction Industry Institute. The University of Texas at Austin, April 2012.

Henneman, E. and Gawlinski, A. (2004). "A Near-Miss Model for Describing the Nurse's Role in the Recovery of Medical Errors,” Journal of Professional Nursing, Elsevier, 20(3) 196 - 201.

Hinze, J. and Godfrey, R. (2003). "An Evaluation of Safety Performance Measures for Construction Projects," Journal of Construction Research, World Scientific, 4(1) 5 - 15.

Marks, E. and Teizer, J. (2012). "Safety First: Real-Time Proactive Equipment Operator and Ground Worker Warning and Alert System in Steel Manufacturing”, Iron \& Steel Technology, AIST, 9(10), pp. 56-69.

Pratt, S., Fosbroke, D., and Marsh, S.M. (2001). "Building Safer Highway Work Zones: Measures to Prevent Worker Injuries from Vehicles and Equipment." Department of Health and Human Services, 1(1): 5-6.

Teizer, J., Allread, B.S., Fullerton, C.E., and Hinze, J. (2010). “Autonomous Pro-Active Real-time Construction Worker and Equipment Operator Proximity Safety Alert System”, Automation in Construction, 19(5): 630-640. 